\title{
Development of a New Autophagosome Sensor With an LC3-interacting Region (LIR) Motif and a Hydrophobic Domain
}

\author{
Yang Hoon Huh ${ }^{1,5}$, Yu-Kyung Lee ${ }^{2,5}$, Yong-Woo Jun ${ }^{3,5}$, Su-Min Um³ ${ }^{3}$ Bong-Kiun Kaang ${ }^{4 *}$, \\ Deok-Jin Jang ${ }^{3, *}$, and Jin-A Lee ${ }^{2, *}$ \\ 1. Nano-Bio EM Research Group, Korea Basic Science Institute, 169-148 Gwahak-ro, Yuseong-gu, \\ Daejeon, Korea. \\ 2. Department of Biological Sciences and Biotechnology, College of Life Sciences and Nanotechnology, \\ Hannam University, Daejeon, Korea. \\ 3. Department of Applied Biology, College of Ecology and Environment, Kyungpook National \\ University, Sang-ju, Korea. \\ 4. Department of Biological Sciences, College of Natural Sciences, Seoul National University, Seoul, \\ Korea. \\ 5. These authors contributed equally to this work.
}

Macroautophagy, hereafter referred to as autophagy, is an evolutionarily conserved lysosomal degradation pathway for long-lived proteins, organelles, or certain cytosolic components such as RNA, or lipids [1]. Although autophagy is primarily considered a kind of a cellular defense mechanism for the provision of nutrients via self-digestion and protection of cells during starvation [2, 3], a growing body of evidence points to autophagy's important roles in many other physiological processes, such as regulation of cellular homeostasis, differentiation, or metabolism [4]. Formation or degradation of the autophagosome, a hallmark of autophagy, is necessary for the whole autophagic process. Therefore, monitoring of autophagosomes is the most important part of autophagy research. To date, overexpression of green fluorescent protein (GFP)/red fluorescent protein (RFP)-light chain 3 (LC3), with specific localization to the autophagosome, has been commonly used for research in this field, but has some limitations due to artifacts related to overexpression of LC3. Here, we developed a new probe for autophagosomes that detects endogenous LC3 in the autophagosome using an LC3-interacting region (LIR(Fy)) motif from FYVE and coiled-coil domain containing 1 (FYCO1) and an N-terminal hydrophobic domain (S(N20)) from Aplysia phosphodiesterase 4 (ApPDE4) short-form. Although either LIR(Fy)-EGFP or S(N20)-EGFP alone was barely localized to the autophagosome, when S(N20) was fused to LIR(Fy)-EGFP, the resulting S(N20)-LIR(Fy)-EGFP was efficiently localized to endogenous LC3-positive autophagosomes induced by either starvation or rapamycin treatment in wild-type (WT) mouse embryonic fibroblasts (MEFs) but not in atg5 $\%$ MEFs. Our immunogold-electron-microscopic experiments confirmed that S(N20)-LIR(Fy)-EGFP was preferentially localized to the autophagosome in WT MEFs but not atg5 ${ }^{-}$MEFs. Finally, we demonstrated that S(N20)-LIR(Fy)-EGFP could detect autophagosomes accumulated in poly $(\mathrm{Q})$-expanded-mutant-huntingtin-expressing neurons; this finding points to possible applications to disease models based on autophagy. Thus, we propose a novel autophagosome sensor for live cells, which is expected to be widely used in autophagy research and practical applications [5].

References:

[1] Y Feng et al., Cell Res. 24 (2014), p. 24-41.

[2] U Pfeifer, Virchows Arch. B. Cell Pathol. 12 (1973), p. 195-211.

[3] DJ Klionski, Nat. Rev. Mol. Cell Biol. 8 (2007), p. 931-937.

[4] DJ Klionski, J. Innate Immun. 5 (2013), p. 427-433. 
[5] This work was supported by the National Honor Scientist Program through a grant to B.-K.K. and by National Research Foundation (grant \# 2014R1A1A4A01003859) and Korea Health Technology R\&D project (\# HI14C1891) to J.-A L. and by National Research Foundation (2014-R1A1A2012804) to D.J.J and by KBSI grant (\#36700).

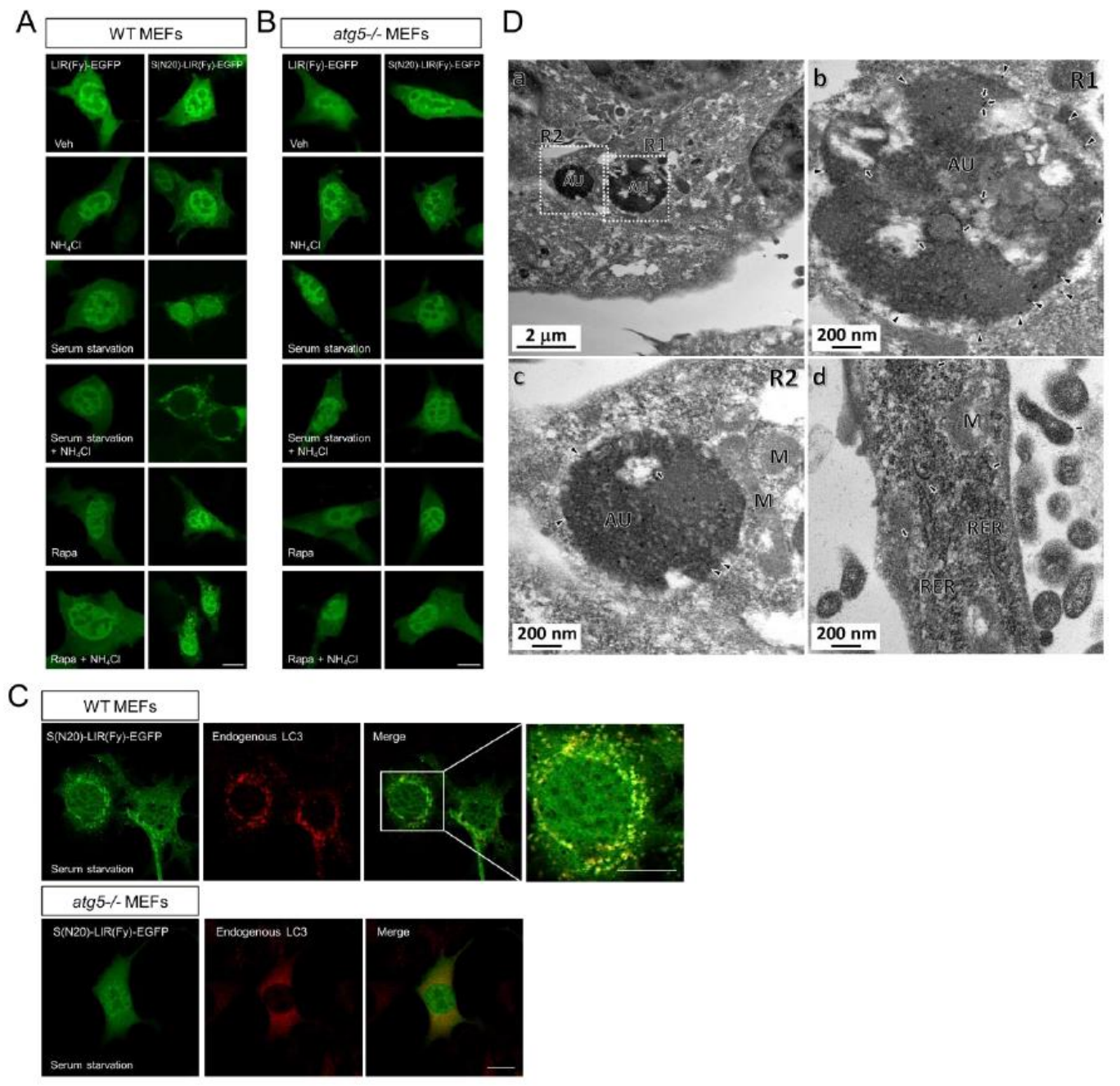

Figure 1. Effective localization of S(N20)-LIR(Fy)-EGFP to autophagosome structures during autophagy induction with serum starvation or rapamycin in an autophagy-dependent manner. A vector encoding LC3-interacting region (LIR(Fy))-EGFP or S(N20)-LIR(Fy)-EGFP was transfected into WT or atg $5^{-1-}$ MEFs. For autophagy induction, the cells were incubated with $10 \mathrm{nM}$ rapamycin for $4 \mathrm{~h}$ or starved (serum deprivation) for $24 \mathrm{~h}$ in the presence or absence of $\mathrm{NH}_{4} \mathrm{Cl}(10 \mathrm{mM})$. (A-B) Confocal images showing the localization of S(N20)-LIR(Fy)-EGFP to autophagic vacuoles during autophagy induction in WT MEFs (A) or atg $5^{-/}$MEFs (B). (C) Confocal images showing the localization of S(N20)-LIR(Fy)-EGFP to endogenous LC3-positive autophagosome detected by anti-LC3 antibody during autophagy induction in WT MEFs (upper) or atg $5^{-1}$ MEFs (lower). Scale bar, $10 \mu \mathrm{m}$. (D) Cell images showing immunogold labeling of LC3-bound GFP in autophagic vacuoles (AU) in WT MEFs (a-c: an enlarged view of R1 and R2) or in atg $5^{-}$MEFs (d) during autophagy induction with serum starvation. Arrowheads indicate immunogold labeling of LC3-bound GFP in the autophagosomal membrane, whereas the arrows show LC3 inside autophagosomes. R: region, M: mitochondrion, RER: rough endoplasmic reticulum. Veh, vehicle; Rapa, rapamycin. 